\title{
ON PARTITIONING AND PACKING PRODUCTS WITH RECTANGLES
}

\author{
Rudolf Ahlswede And Ning Cai
}

Universität Bielefeld

Fakultät für Mathematik

Postfach 100131

33501 Bielefeld

Germany 


\section{ABSTRACT}

In [1] we introduced and studied for product hypergraphs $\mathcal{H}^{n}=\prod_{i=1}^{n} \mathcal{H}_{i}$, where $\mathcal{H}_{i}=\left(\mathcal{V}_{i}, \mathcal{E}_{i}\right)$, the minimal size $\pi\left(\mathcal{H}^{n}\right)$ of a partition of $\mathcal{V}^{n}=\prod_{i=1}^{n} \mathcal{V}_{i}$ into sets that are elements of $\mathcal{E}^{n}=\prod_{i=1}^{n} \mathcal{E}_{i}$.

The main result was that

$$
\pi\left(\mathcal{H}^{n}\right)=\prod_{i=1}^{n} \pi\left(\mathcal{H}_{i}\right)
$$

if the $\mathcal{H}_{i}$ 's are graphs with all loops included. A key step in the proof concerns the special case of complete graphs. Here we show that (1) also holds when the $\mathcal{H}_{i}$ are complete $d$-uniform hypergraphs with all loops included, subject to a condition on the sizes of the $\mathcal{V}_{i}$. We present also an upper bound on packing numbers. 


\section{INTRODUCTION}

For hypergraphs $\mathcal{H}_{i}=\left(\mathcal{V}_{i}, \mathcal{E}_{i}\right) \quad(1 \leq i \leq n)$ we define the product hypergraph $\mathcal{H}^{n}=\left(\mathcal{V}^{n}, \mathcal{E}^{n}\right)=\left(\prod_{i=1}^{n} \mathcal{V}_{i}, \prod_{i=1}^{n} \mathcal{E}_{i}\right)$. Edges of cardinality 1 are called loops. Special hypergraphs are graphs $\mathcal{G}=(\mathcal{V}, \mathcal{E})$, which are defined by the property

$$
|E| \in\{1,2\} \text { for all } E \in \mathcal{E}
$$

and, more generally, $d$-uniform hypergraphs (with or without loops), which satisfy

$$
|E| \in\{1, d\} \text { for all } E \in \mathcal{E} \text {. }
$$

In particular there are $d$-uniform hypergraphs with all loops included, that is, $\{\{v\}$ : $v \in \mathcal{V}\} \subset \mathcal{E}$.

When the set $\left(\begin{array}{l}\mathcal{V} \\ d\end{array}\right)$ of all vertex sets of cardinality $d$ is contained in the edge set $\mathcal{E}$, then we speak of a complete $d$-uniform hypergraph.

In [1] we introduced the partition number $\pi(\mathcal{H})$ as the minimal size of a partition of $\mathcal{V}$ into sets that are members of $\mathcal{E}$, if a partition exists, and as $\infty$ otherwise. When $\mathcal{G}_{i}=\left(\mathcal{V}_{i}, \mathcal{E}_{i}\right) \quad(i=1,2, \ldots, n)$ are arbitrary finite graphs with all loops included, then we have obviously for the partition number $\pi\left(\mathcal{G}_{i}\right)=\left|\mathcal{V}_{i}\right|-\nu\left(\mathcal{G}_{i}\right)$, where $\nu\left(\mathcal{G}_{i}\right)$ is the matching number of $\mathcal{G}_{i}$. A discovery of [1] is that for the hypergraph product $\mathcal{H}^{n}=\mathcal{G}_{1} \times \cdots \times \mathcal{G}_{n}$

$$
\pi\left(\mathcal{H}^{n}\right)=\prod_{i=1}^{n} \pi\left(\mathcal{G}_{i}\right)
$$

An important step in our proof is to show (1.3) when all $\mathcal{G}_{i}$ 's are complete.

Here we establish the following generalization.

\section{Theorem.}

For complete $d$-uniform hypergraphs with all loops $\mathcal{H}_{i}=\left(\mathcal{V}_{i}, \mathcal{E}_{i}\right)$, that is, $\mathcal{E}_{i}=$ $\left(\begin{array}{c}\mathcal{V}_{i} \\ d\end{array}\right) \cup\left\{\{v\}: v \in \mathcal{V}_{i}\right\} \quad(i=1,2, \ldots, n)$ write $\left|\mathcal{V}_{i}\right|=d q_{i}+r_{i}, 0 \leq r_{i}<d$.

Then for $\mathcal{H}^{n}=\prod_{i=1}^{n} \mathcal{H}_{i}$ satisfying

$(*) \quad d>\prod_{i: r_{i} \neq 0} r_{i}$

we have

$$
\pi\left(\mathcal{H}^{n}\right)=\prod_{i=1}^{n} \frac{\left|\mathcal{V}_{i}\right|+(d-1) r_{i}}{d}=\prod_{i=1}^{n}\left(q_{i}+r_{i}\right)=\prod_{i=1}^{n} \pi\left(\mathcal{H}_{i}\right)
$$

(Theorem 2' of [1] is covered by the case $d=2$ ). 
We present also an upper bound on packing numbers, the maximal number of disjoint edges, in Section 5.

\section{Remarks.}

1. The condition $\left(^{*}\right)$ cannot be omitted. In Example 1 of [1] we have $\left|\mathcal{V}_{i}\right|=7$, $n=2, d=4, r=3, q=1$, and thus $d<r^{2}$ in violation of $(*)$. However, $\pi\left(\mathcal{H}^{2}\right) \leq 13<(1+3)^{2}=16$ and thus (1.1) does not hold.

2. The Theorem always applies, if $r_{i} \in\{0,1\}$ for all $i$. In particular (1.1) holds for complete $d$-uniform hypergraphs including all loops, if $|\mathcal{V}| \in\{d q, d q+1\}$.

3. In the light of our former results for graphs with all loops included it is natural to study products of hypergraphs $\mathcal{H}_{i}=\left(\mathcal{V}_{i}, \mathcal{E}_{i}\right)$, whose edge sets $\mathcal{E}_{i}$ are downsets, that is, for all $i$ and $E \in \mathcal{E}_{i}$

$$
F \subset E \text { implies } F \in \mathcal{E}_{i} \text {. }
$$

In spite of several attempts we could not even settle the case

$$
\mathcal{E}_{i}=\left\{E \subset \mathcal{V}_{i}:|E| \leq d_{i}\right\}
$$

An immediate technical difficulty is caused by the fact that there may be nonisomorphic minimal partitions of $\mathcal{H}_{i}$.

For example in the case $\mathcal{V}_{i}=\{0,1, \ldots, 8\}$ and $d=4$, both,

$$
\{\{0,1,2,3\},\{4,5,6,7\},\{8\}\} \text { and }\{\{0,1,2,3\},\{4,5,6\},\{7,8\}\} \text { are optimal. }
$$

For products of such hypergraphs there is a lot of freedom for minimal partitions.

The paper is organized as follows:

In Section 2 we prove an present as Lemma 1 our key identity.

Two simple consequences, Lemma 2 and Lemma 3, are derived in Section 3.

Section 4 contains the proof of the Theorem.

Finally, in Section 5 we use Lemma 3 to derive a bound on packing numbers (Corollary 1) and to determine the packing number of products of two graphs, which have Hamiltonian cycles (Corollary 2).

\section{AN IDENTITY FOR PACKINGS IN PRODUCTS OF GENERAL HYPERGRAPHS}

For $I \subset[n]$, we write $\mathcal{H}^{I}=\prod_{i \in I} \mathcal{H}_{i}, \mathcal{V}^{I}=\prod_{i \in I} \mathcal{V}_{i}, \mathcal{E}^{I}=\prod_{i \in I} \mathcal{E}_{i}$, and for given $E^{n}=\prod_{i=1}^{n} E_{i}$ and $v^{n}=\prod_{i=1}^{n} v_{i}$ we also write $E^{I}=\prod_{i \in I} E_{i}$ and $v^{I}=\prod_{i \in I} v_{i}$, respectively.

With a packing $\mathcal{P}$ in $\mathcal{H}^{n}$ we associate the support 


$$
\mathcal{S}(\mathcal{P})=\bigcup_{E^{n} \in \mathcal{P}} E^{n}
$$

Also, for $I \subset[n], v^{I} \in \mathcal{V}^{I}$ and $\mathcal{P}$ we introduce the packing of $\mathcal{H}^{I^{c}}$

$$
\mathcal{P}_{v^{I}}=\left\{E^{I^{c}}: E^{n} \in \mathcal{P} \text { with } v^{I} \in E^{I}\right\}
$$

Geometrically this means taking a "slice" from the product (corresponding to fixing some values in the coordinates I) and looking at the restrictions of all the sets in the packing to that slice.

Finally, for general functions $\varphi_{i}: \mathcal{E}_{i} \rightarrow \mathbb{R}(i=1,2, \ldots, n), J \subset[n]$ and $E^{n}$ we set

$$
\varphi\left(E^{J}\right)=\prod_{i \in J} \varphi_{i}\left(E_{i}\right)
$$

and for $\mathcal{F} \subset \mathcal{E}^{J}$ we set

$$
\varphi(\mathcal{F})=\sum_{E^{J} \in \mathcal{F}} \varphi\left(E^{J}\right)
$$

$(\varphi(\phi)=0$ by convention).

We are now ready to state our basic identity.

Lemma 1. For every packing $\mathcal{P}$ of the product hypergraph $\mathcal{H}^{n}$ and every function $\varphi$ defined by (2.3) and (2.4)

$$
\sum_{I \subset[n]} \sum_{v^{I}} \varphi\left(\mathcal{P}_{v^{I}}\right)=\sum_{E^{n} \in \mathcal{P}} \prod_{\ell=1}^{n}\left(\left|E_{\ell}\right|+\varphi_{\ell}\left(E_{\ell}\right)\right) .
$$

\section{Proof:}

$$
\begin{aligned}
\sum_{I \subset[n]} \sum_{v^{I}} \varphi\left(\mathcal{P}_{v^{I}}\right) & =\sum_{I \subset[n]} \sum_{v^{I}} \sum_{E^{I^{c} \in \mathcal{P}_{v^{I}}}} \varphi\left(E^{I^{c}}\right)=\sum_{I \subset[n]} \sum_{E^{n} \in \mathcal{P}} \sum_{v^{I} \in E^{I}} \varphi\left(E^{I^{c}}\right) \\
& =\sum_{I \subset[n]} \sum_{E^{n} \in \mathcal{P}}\left|E^{I}\right| \varphi\left(E^{I^{c}}\right)=\sum_{E^{n} \in \mathcal{P}} \sum_{I \subset[n]}\left(\prod_{i \in I}\left|E_{i}\right|\right)\left(\prod_{j \in I^{c}} \varphi_{j}\left(E_{j}\right)\right) \\
& =\sum_{E^{n} \in \mathcal{P}} \prod_{\ell=1}^{n}\left(\left|E_{\ell}\right|+\varphi_{\ell}\left(E_{\ell}\right)\right) \quad \text { (by the Binomial formula). }
\end{aligned}
$$

In the next section we derive two consequences of Lemma 1. The first is used in the proof of the Theorem. The second is used for our results on packings in Section 5.

In [4] we have developed a more general machinery to study partitions of products of hypergraphs. We employ there Rota's theorem of Möbius transforms for posets (see [2] or [3]). However, the best concrete results there are covered also by [1] and the present result. 


\section{Two CONSEQUenCES OF Lemma 1}

\section{Lemma 2.}

Let $\mathcal{H}^{n}$ be a product of $d$-uniform hypergraphs with loops, then for a partition $\mathcal{P}$ of $\mathcal{H}^{n}$

$$
d^{n}|\mathcal{P}|=\sum_{I \nsubseteq[n], v^{I} \in \mathcal{V}^{I}}(d-1)^{n-|I|} J_{v^{I}}+\prod_{i=1}^{n}\left|\mathcal{V}_{i}\right|
$$

where $J_{v^{I}}$ is the number of elements with size 1 in the partition $\mathcal{P}_{v^{I}}$ of $\mathcal{V}^{I^{c}}$.

\section{Lemma 3.}

For a packing $\mathcal{P}$ in $\mathcal{H}^{n}=\prod_{i=1}^{n} \mathcal{H}_{i}$, where $\mathcal{H}_{i}$ is a $d_{i}$-uniform hypergraph without loops,

$$
|\mathcal{P}|=\frac{\sum_{0<|I|<n} \sum_{v^{I}}\left|\mathcal{P}_{v^{I}}\right|}{\prod_{i=1}^{n}\left(d_{i}+1\right)-\left(1+\prod_{i=1}^{n} d_{i}\right)}
$$

Proof of Lemma 2: We have for all non-singletons $E_{i}$ that $\left|E_{i}\right|=d$. Choose $\varphi_{i}\left(E_{i}\right)=d-\left|E_{i}\right|$. Then the LHS of (2.5) is

$$
\sum_{I \subset[n]} \sum_{v^{I}} \varphi\left(\mathcal{P}_{v^{I}}\right)=\sum_{I \subset[n]} \sum_{v^{I} \in \mathcal{V}^{I}}(d-1)^{n-|I|} J_{v^{I}}
$$

where $J_{v^{I}}$ is the number of singletons in $\mathcal{P}_{v^{I}}$, and the RHS of $(2.5)$ is

$$
\sum_{E^{n} \in \mathcal{P}} \prod_{\ell=1}^{n}\left(\left|E_{\ell}\right|+d-\left|E_{\ell}\right|\right)=d^{n}|\mathcal{P}|
$$

Since $\mathcal{P}$ is a partition, we have $\sum_{v^{[n]}} J_{v^{[n]}}=\prod_{i=1}^{n}\left|\mathcal{V}_{i}\right|$ and thus (3.1).

Proof of Lemma 3: Choose now $\varphi_{i}\left(E_{i}\right)=1$ for all $E_{i} \in \mathcal{E}_{i}$ and $i=1,2, \ldots, n$. Then (2.5) becomes

$$
\sum_{I \subset[n]} \sum_{v^{I}}\left|\mathcal{P}_{v^{I}}\right|=\prod_{i=1}^{n}\left(d_{i}+1\right)|\mathcal{P}|
$$

Since $\mathcal{P}_{\phi}=\mathcal{P}$ and $\left|\mathcal{P}_{b^{n}}\right|=1$, if $b^{n} \in \mathcal{S}(\mathcal{P})$, and equal to 0 otherwise, we conclude

$$
\sum_{0<|I|<n} \sum_{v^{I}}\left|\mathcal{P}_{v^{I}}\right|=\prod_{i=1}^{n}\left(d_{i}+1\right)|\mathcal{P}|-|\mathcal{P}|-|\mathcal{S}(\mathcal{P})|
$$


As $|\mathcal{S}(\mathcal{P})|=\prod_{i=1}^{n} d_{i}|\mathcal{P}|$ we have established the result.

\section{Proof of TheOrem}

We show first that condition $(*)$ implies

$$
J_{v^{I}} \geq \prod_{j \in I^{c}} r_{j} \text { for } I \subset[n]
$$

Suppose that for some $I$ the inequality does not hold: $\prod_{j \in I^{c}} r_{j}-J_{v^{I}}>0$. Since every $E^{n} \in \prod_{i=1}^{n}\left\{\{v\}: v \in \mathcal{V}_{i}\right\}$ has size 1 and every $E^{n} \in \prod_{i=1}^{n}\left(\mathcal{E}_{i} \cup\{\{v\}: v \in\right.$ $\left.\left.\mathcal{V}_{i}\right\}\right) \backslash \prod_{i=1}^{n}\left\{\{v\}: v \in \mathcal{V}_{i}\right\}$ has a size divisible by $d$, we can write

$$
\prod_{j \in I^{c}}\left|\mathcal{V}_{j}\right|=A d+\prod_{j \in I^{c}} r_{j}=B d+J_{v^{I}}
$$

where $A$ and $B$ are positive integers, and therefore $d \mid\left(\prod_{j \in I^{c}} r_{j}-J_{v^{I}}\right)$ in contradiction to our supposition and $(*)$.

Now (4.1) and Lemma 2 imply

$$
\begin{aligned}
d^{n}|\mathcal{P}| & \geq \sum_{k=0}^{n-1}(d-1)^{n-k} \sum_{I:|I|=k} \prod_{i \in I}\left|\mathcal{V}_{i}\right| \prod_{j \in I^{c}} r_{j}+\prod_{i=1}^{n}\left|\mathcal{V}_{i}\right| \\
& =\prod_{i=1}^{n}\left[\left|\mathcal{V}_{i}\right|+(d-1) r_{i}\right] \quad \text { (by the Binomial formula) and hence }|\mathcal{P}| \geq \prod_{i=1}^{n}\left(q_{i}+r_{i}\right) .
\end{aligned}
$$

The opposite inequality follows from the fact that the product of partitions is a partition.

\section{A CONSEQUence of Lemma 2}

Denote by $p(\mathcal{H})$ the packing number of $\mathcal{H}$.

Corollary 1. For $d_{i}$-uniform hypergraphs $\mathcal{H}_{i}=\left(\mathcal{V}_{i}, \mathcal{E}_{i}\right) \quad(i=1,2)$

$$
p\left(\mathcal{H}_{1} \times \mathcal{H}_{2}\right) \leq\left\lfloor\frac{\left|\mathcal{V}_{1}\right| p\left(\mathcal{H}_{2}\right)+\left|\mathcal{V}_{2}\right| p\left(\mathcal{H}_{1}\right)}{d_{1}+d_{2}}\right\rfloor
$$

We present finally a striking example, which shows that (5.1) is surprisingly sharp. 
Corollary 2. For two graphs $\mathcal{G}_{i}=\left(\mathcal{V}_{i}, \mathcal{E}_{i}\right) \quad(i=1,2)$ with $\left|\mathcal{V}_{1}\right|=\left|\mathcal{V}_{2}\right|=u$, both having Hamiltonian cycles,

$$
p\left(\mathcal{G}_{1} \times \mathcal{G}_{2}\right)=\left\lfloor\frac{u\left\lfloor\frac{u}{2}\right\rfloor}{2}\right\rfloor .
$$

Proof: The upper bound on $p\left(\mathcal{G}_{1} \times \mathcal{G}_{2}\right)$ follows from Corollary 1 . The opposite inequality is trivial for even $m$ and follows for odd $m$ by inspection (Notice that deleting edges can only make the packings worse!) of configurations, which we now describe.

We label the edges of $m$-cycles $C_{m}$ by $0,1, \ldots, m-1$, such that any two connected edges have labels with difference $1(\bmod m)$, and denote by $\oplus$ and $\ominus$ the addition and subtraction in the modulo $m$ group.

Case: $m=4 k+1(k \geq 1)$. Here (5.1) yields $P\left(C_{4 k+1}^{2}\right) \leq k(4 k+1)$.

Tightness of this bound follows from consideration of the packing

$$
\mathcal{P}=\{(i, 2 i \oplus 4 j): 0 \leq i \leq 4 k, 0 \leq j \leq k-1\}
$$

Case: $m=4 k+3$. Here (5.1) implies $P\left(C_{k+3}\right) \leq 4 k^{2}+5 k+1$

and tightness of this bound follows from the consideration of the construction $\mathcal{P}^{\prime}=$ $\bigcup_{i=0}^{m-1} \mathcal{P}_{i}$, where

$$
\mathcal{P}_{i}= \begin{cases}\left\{\left(i, 2 j \ominus \frac{i}{2}\right): j=0,1, \ldots, k\right\}, & \text { if } i \text { is even and } i \neq 4 k+2, \\ \left\{\left(i, 2 j \ominus \frac{i}{2}\right): j=0,1, \ldots, k-1\right\}, & \text { if } i=4 k+2, \\ \left\{\left(i, 2 j \ominus \frac{i-1}{2}\right): j=k+1, \ldots, 2 k\right\}, & \text { if } i \text { is odd. }\end{cases}
$$

\section{REFERENCES}

[1] R. Ahlswede and N. Cai, On extremal set partitions in Cartesian product spaces, Combinatorics, Probability \& Computing 2, 211-220, 1993.

[2] G.C. Rota, On the foundations of combinatorial theory I. Möbius functions, Z. Wahrscheinlichkeitstheorie 2, 340-368, 1964.

[3] R.P. Stanley, Enumerative Combinatorics, Vol. I, Wadsworth \& Brooks/Coll, Advanced Books \& Software, Monterey, California 1986.

[4] R. Ahlswede and N. Cai, On poset partitions and hypergraph products, Preprint 93-008 of SFB 343, Diskrete Strukturen in der Mathematik, Bielefeld, March 1993. 